\title{
Association of Sleep Quality with Cardiorespiratory Fitness in Male High School Students
}

\author{
Seung-Taek Lim ${ }^{1,2,3}$. Eunjae Lee Le,3 $^{2,4}$ \\ Received: 4 February 2020 / Accepted: 25 February 2020 /Published online: 12 March 2020 \\ (C) Dr. K C Chaudhuri Foundation 2020
}

To the Editor: Researchers generally assume that sleep has three main functions. These include 1) Restoring energies (recovery from daytime activities), 2) Protection from potential danger (keeping us quiet at night when our sensory capacities are limited), and 3) Learning (consolidation of important memories) [1]. Given that we spend about a one-third of our entire life sleeping, sleep affects our development, our daily functions, and important functions related to our health [2]. A possible causal link between sleep deprivation and cardio-metabolic risk is strengthened by the evidence obtained from the cohort in the study by King et al. [3]. Thus, in high school students, there is a significant relationship of cardiopulmonary endurance and sleep quality.

We investigated the physical fitness and sleep quality of male high school students participating in regular physical activities. Even though the participation was regular in the physical activities, a negative correlation was found between the Pittsburgh Sleep Quality Index (PSQI) global score and $\mathrm{VO}_{2}$ max; and PSQI global score, PSQI sleep quality, PSQI sleep onset latency, PSQI sleep duration, and PSQI daytime dysfunction showed significant differences between the quality sleep group and poor quality group. One possibility is that increased chemosensory receptor reflex sensitivity in critical components during the respiratory cycle of sleep disorders involves a long-term response with low ventilation, reduced cardiac output, small lung volume, and large slopes between atmospheric and alveolar $\mathrm{CO}_{2}$ tension [4]. Support exists for the increasing evidence of an association between shortened

Eunjae Lee

eunjaelee0421@gmail.com

1 Institute of Sport Science, Kangwon National University, Chuncheon-si, Gangwon-do, Republic of Korea

2 Waseda Institute for Sport Sciences, Waseda University, Saitama, Japan

3 Nasaret International Hospital, Incheon, Republic of Korea

4 Center for Sport Science in Incheon, Incheon, Republic of Korea sleep duration, longer sleep onset latency, daytime dysfunction and/or poor sleep quality even if an individual participates in regular physical activity.

A combination of negative lifestyle behavior, lack of physical activity and sleep, and poor physical fitness is a major contributor to cardiovascular health complications in at-risk adolescents [5]. Thus, sleep quality and cardiopulmonary endurance in adolescence are important and should be improved.

Association between poor sleep quality and lower cardiopulmonary endurance, and shortened sleep duration, longer sleep onset latency, and daytime dysfunction leads to poor sleep quality even if one participates in regular physical activity. Therefore, this study provides preliminary evidence that adolescents with poor sleep quality must continue to engage in exercise or physical activity to improve cardiopulmonary endurance.

\section{Compliance with Ethical Standards}

Conflict of Interest None.

\section{References}

1. Gerber M, Brand S, Holsboer-Trachsler E, Pühse U. Fitness and exercise as correlates of sleep complaints: is it all in our minds? Med Sci Sports Exerc. 2010;42:893-901.

2. Morin CM, Bootzin RR, Buysse DJ, Edinger JD, Espie CA, Lichstein KL. Psychological and behavioral treatment of insomnia: update of the recent evidence (1998-2004). Sleep. 2006;29:1398-414.

3. King CR, Knutson KL, Rathouz PJ, Sidney S, Liu K, Lauderdale DS. Short sleep duration and incident coronary artery calcification. JAMA. 2008;300:2859-66.

4. Francis DP, Willson K, Davies LC, Coats AJ, Piepoli M. Quantitative general theory for periodic breathing in chronic heart failure and its clinical implications. Circulation. 2000;102:2214-21.

5. Countryman AJ, Saab PG, Llabre MM, Penedo FJ, McCalla JR, Schneiderman N. Cardiometabolic risk in adolescents: associations with physical activity, fitness, and sleep. Ann Behav Med. 2013;45:121-31.

Publisher's Note Springer Nature remains neutral with regard to jurisdictional claims in published maps and institutional affiliations. 